\title{
ON HOMEOMORPHISMS OF THE PLANE, AND THEIR EMBEDDING IN FLOWS
}

\author{
BY S. A. ANDREA ${ }^{1,2}$
}

Communicated by G. A. Hedlund, October 26, 1964

Let $T$ be an orientation-preserving homeomorphism of the plane $E^{2}$ onto itself. Suppose that $T$ has no fixed points, i.e., that on the sphere $S^{2}=E^{2} \cup\{\infty\}, T$ has no fixed point other than $\infty$. Then, by the Brouwer Translation Theorem [1], one can construct a Jordan curve $\Gamma \subset S^{2}$ in such a manner that $\Gamma \cap T \Gamma=\{\infty\}$. If $p$ is a preassigned point in $E^{2}$, then $\Gamma$ can be made so that $p$ lies between $\Gamma$ and $T \Gamma$.

In the present note we state two new results (Lemma 1 and Theorem 1) about the homeomorphisms treated in Brouwer's theorem. The proofs, which depend to some extent upon Brouwer's techniques, will appear elsewhere. Having presented these results, we will apply them to the problem of embedding homeomorphisms in flows. Indeed, we obtain a natural characterization of those homeomorphisms of the plane which are equivalent to translations.

LEMMA 1. Let $T$ be an orientation-preserving homeomorphism, without fixed points, of $E^{2}$ onto itself. Let $C \subset E^{2}$ be compact and connected. If $C \cap T C=0$, then $C \cap T^{n} C=0$ for all $n \neq 0$.

REMARK. This lemma can be used to simplify some of the proofs in Brouwer's original paper.

The second result, like the Translation Theorem, shows how arbitrary homeomorphisms resemble the special homeomorphism $(x, y)$ $\rightarrow(x+1, y)$.

THEOREM 1. Let $T$ be a homeomorphism of $E^{2}$ onto itself. Suppose that $T$ preserves the sense of orientation and has no fixed points. Then for any compact set $A \subset E^{2}$ there is an unbounded connected set $B \subset E^{2}$ which does not meet $\mathrm{U}_{-\infty}^{+\infty} T^{n} A$.

REMARK. One can show by examples that the conclusion of the theorem is false if either of the two assumptions is dropped, or if $E^{2}$ is replaced by $E^{1}, E^{3}, E^{4}, \cdots$.

1 This research has been supported in part by the U. S. Army Research Office (Durham).

2 The author also wishes to express his thanks to Professor F. Brock Fuller for his guidance during the preparation of this work. 
We turn now to the question of whether an arbitrary homeomorphism $T$ can be embedded in some one-parameter group of homeomorphisms $\left\{T^{\alpha}\right\},-\infty<\alpha<\infty$. Such a group is called a flow: $T^{\alpha}\left(T^{\beta} p\right)=T^{\alpha+\beta} p$ in general, and the point $T^{\alpha} p$ is jointly continuous in the real number $\alpha$ and the point $p$. We restrict ourselves to the homeomorphisms described in Brouwer's theorem.

Our method will be to study certain subsets of $E^{2}$ that would have to be left invariant by a flow in which $T$ were embedded. The following lemma, a direct consequence of Lemma 1 , will enable us to define these subsets.

LEMMA 2 (BROUWER). Let $T$ be a homeomorphism of $E^{2}$ onto itself which preserves orientation and has no fixed points. Then, for any $p \in E^{2}$, we have $T^{n} p \rightarrow \infty$ as $n \rightarrow \pm \infty$.

Proof. If the lemma were false we would have $T^{n_{k}} p \rightarrow q$ for some $q \in E^{2}$ and some sequence of integers $\left\{n_{k}\right\}$ with $\left|n_{k}\right| \rightarrow \infty$. But $q$ is contained in a small disc $D$ which does not meet its image $T D$. By Lemma $1, D$ does not meet $T^{n} D$ for any $n \neq 0$, and, hence, the sequence $\left\{T^{n_{k}} p\right\}$ can have at most one representative in $D$. This is a contradiction of the statement that $T^{n_{k}} p \rightarrow q$. I

Now let $\left\{E_{n}\right\},-\infty<n<\infty$, be any sequence of sets. We say that the sequence diverges, written " $E_{n} \rightarrow \infty$ as $n \rightarrow \pm \infty$," if, given any compact set $A \subset E^{2}$, the intersection $E_{n} \cap A$ is void for sufficiently large $|n|$.

Our invariant subsets will be the equivalence classes from the following relation: if $p \in E^{2}$ and $q \in E^{2}$ we say that $p$ is co-divergent with $q$ if $p$ and $q$ are endpoints of some curve segment $\Lambda$ for which $T^{n} \Lambda \rightarrow \infty$ as $n \rightarrow \pm \infty$.

The assertion that co-divergence is a reflexive relation is precisely the assertion of Lemma 2. Symmetry and transitivity are easy to verify.

The "fundamental regions" of the homeomorphism $T$ are just the equivalence classes of co-divergent points.

It is now easy to prove

Lemma 3. Let $T$ be a homeomorphism of $E^{2}$ onto itself which preserves orientation and has no fixed points. If $T$ is embedded in the flow $\left\{T^{\alpha}\right\}$, then each fundamental region of $T$ is mapped onto itself by each homeomorphism $T^{\alpha}$.

Proof. Observe first that if $\left\{\alpha_{n}\right\}$ is any sequence of real numbers, with $\left|\alpha_{n}\right| \rightarrow \infty$ as $n \rightarrow \pm \infty$, then $T^{\alpha_{n}} p \rightarrow \infty$ as $n \rightarrow \pm \infty$. To see this, let $A$ be any compact set in $E^{2}$. The set $B=\bigcup_{-1 \leq \alpha_{\leq} 0} T^{\alpha} A$ is also com- 
pact. Write $m_{n}=\left[\alpha_{n}\right]$, the greatest integer contained in $\alpha_{n}$; then $T^{\alpha_{n}} p \in A$ implies that $T^{m_{n}} p \in B$ and hence can occur for at most finitely many $n$.

By our observation, the curve $\Gamma_{\beta}=\left\{T^{\beta t} p: 0 \leqq t \leqq 1\right\}$ satisfies the condition that $T^{n} \Gamma_{\beta} \rightarrow \infty$ as $n \rightarrow \pm \infty$. Hence, $p$ is co-divergent with $T^{\beta} p$ for any $p \in E^{2},-\infty<\beta<\infty$, and we have proved Lemma 3.I

By constructing homeomorphisms whose fundamental regions are badly behaved, one can obtain counterexamples to the conjecture that any homeomorphism satisfying the hypotheses of Brouwer can be embedded in a flow. On the other hand, Theorem 1 can be used to prove the following positive result:

THEOREM 2. Let $T$ be a homeomorphism of $E^{2}$ onto itself which preserves orientation and has no fixed points. If $T$ has exactly one fundamental region, that being $E^{2}$ itself, then $T$ is equivalent to a translation, i.e., there exists a homeomorphism $U$ of $E^{2}$ onto itself such that $U T U^{-1}$ is just the isometry $(x, y) \rightarrow(x+1, y)$.

The first step in proving Theorem 2 is to show that $T^{n} A \rightarrow \infty$ as $n \rightarrow \pm \infty$ for any compact set $A \subset E^{2}$. It is here that one needs Theorem 1, together with the assumption that $T$ has only one fundamental region. The next and last step is to apply a result of Sperner [2] to get the desired coordinate change $U$.

Detailed proofs of these results will appear elsewhere, along with other theorems about fundamental regions and the behavior of $T$ within them.

\section{REFERENCES}

1. L. E. J. Brouwer, Beweis des ebenen Translationssatzes, Math. Ann. 72 (1912), 37-54.

2. E. Sperner, Hamburger Mathematische Einzelschriften 14 (1933), 1-47.

California Institute of Technology 